\title{
ON AN EXPRESSION OF THE ELECTROMAGNETIC FIELD DUE TO ELECTRONS BY MEANS OF TWO SCALAR POTENTIAL FUNCTIONS
}

\author{
By E. T. WhitTaker.
}

[Roceived 11th November, 1903.-Read 12th November, 1903.]

\section{Object of Paper.}

The object of the present paper is to show that when any number of electrons are moving in any manner the functions which define the resulting electrodynamic field, namely, the three components of dielectric displacement in the æther and the three components of the magnetic force at every point of the field, can be expressed in terms of the derivates of two scalar potential functions. (Previous writers have expressed them in terms of a scalar potential function and a vector potential function, which are equivalent to four scalar potential functions.) These two scalar potential functions are explicitly evaluated in terms of the charges and co-ordinates of the electrons. It is then shown that from these results the general functional form of an electrodynamic disturbance due to electrons can be derived.

2. Explanation of Notation, and Summary of previously known Results.

The work of previous writers, so far as it concerns the present investigation and explains the notation used, may be briefly summarized as follows :-

Let $\rho$ be the volume density of electricity at any place and time, and let $v_{x}, v_{y}, v_{z}$ be the components of its velocity, and $c$ the velocity of light in the æther. Let $d_{x}, d_{y}, d_{z}$ be the three components of the dielectric displacement in the æther, and $h_{x}, h_{y}, h_{z}$ the three components of the magnetic force. Then the fundamental equations of electrodynamics may be written in Lorentz's form (the units being suitably chosen):

$$
\begin{gathered}
\frac{\partial \rho}{\partial t}+\frac{\partial\left(\rho v_{x}\right)}{\partial x}+\frac{\partial\left(\rho v_{y}\right)}{\partial y}+\frac{\partial\left(\rho v_{z}\right)}{\partial z}=0 \\
\frac{\partial d_{x}}{\partial x}+\frac{\partial d_{y}}{\partial y}+\frac{\partial d_{z}}{\partial z}=\rho, \\
\frac{\partial h_{x}}{\partial x}+\frac{\partial h_{y}}{\partial y}+\frac{\partial h_{z}}{\partial z}=0
\end{gathered}
$$




$$
\begin{aligned}
c\left(\frac{\partial h_{z}}{\partial y}-\frac{\partial h_{y}}{\partial z}\right) & =\frac{\partial d_{x}}{\partial t}+\rho v_{x}, & c\left(\frac{\partial d_{z}}{\partial y}-\frac{\partial d_{y}}{\partial z}\right) & =-\frac{\partial h_{x}}{\partial t}, \\
c\left(\frac{\partial h_{x}}{\partial z}-\frac{\partial h_{z}}{\partial x}\right) & =\frac{\partial d_{y}}{\partial t}+\rho v_{y}, & c\left(\frac{\partial d_{x}}{\partial z}-\frac{\partial d_{z}}{\partial x}\right) & =-\frac{\partial h_{y}}{\partial t}, \\
c\left(\frac{\partial h_{y}}{\partial x}-\frac{\partial h_{x}}{\partial y}\right) & =\frac{\partial d_{z}}{\partial t}+\rho v_{z}, & c\left(\frac{\partial d_{y}}{\partial x}-\frac{\partial d_{x}}{\partial y}\right) & =-\frac{\partial h_{z}}{\partial t}
\end{aligned}
$$

In place of $d_{x}, d_{y}, d_{z}, h_{x}, h_{y}, h_{z}$, we can define the field by a scalar potential function $\phi$ and three functions $a_{x}, a_{y}, a_{z}$, which are usually regarded as the three components of a vector potential. The quantities $d_{x}, d_{y}, d_{z}, h_{x}, h_{y}, h_{z}$ are given in terms of $\phi, a_{x}, a_{y}, a_{z}$ by the equations

$$
d_{x}=-\frac{1}{c} \frac{\partial a_{x}}{\partial t}-\frac{\partial \phi}{\partial x}, \quad h_{x}=\frac{\partial a_{z}}{\partial y}-\frac{\partial a_{y}}{\partial z}
$$

and four similar equations for $d_{y}, d_{z}, h_{y}, h_{z}$.

The scalar potential and the three components of the vector potential satisfy the system of equations

$$
\begin{gathered}
c^{2} \nabla^{2} \phi-\frac{\partial^{2} \phi}{\partial t^{2}}=-c^{2} \rho, \quad c^{2} \nabla^{2} a_{x}-\frac{\partial^{2} a_{x}}{\partial t^{2}}=-c \rho v_{x}, \\
c^{2} \nabla a_{y}-\frac{\partial^{2} a_{y}}{\partial t^{2}}=-c \rho v_{y}, \quad c^{2} \nabla^{2} a_{z}-\frac{\partial^{2} a_{z}}{\partial t^{2}}=-c \rho v_{z}, \\
\frac{\partial a_{x}}{\partial x}+\frac{\partial a_{y}}{\partial y}+\frac{\partial a_{z}}{\partial z}+\frac{1}{c} \frac{\partial \phi}{\partial t}=0 .
\end{gathered}
$$

For the fundamental case, namely, that in which the field is due to any number of electrons moving in any way, the scalar potential and the three components of the vector potential are given by the equations

$\phi(x, y, z, t)=\Sigma \frac{e c}{4 \pi} \frac{1}{c \bar{r}+\bar{r} \bar{v} \cos (\bar{v}, \bar{r})}, \quad a_{x}(x, y, z, t)=\Sigma \frac{e}{4 \pi} \frac{\bar{v}_{x}}{c \bar{r}+\bar{r} \bar{v} \cos (\bar{v}, \bar{r})}$,

and two similar equations for $a_{y}$ and $a_{z}$, where $e$ is the charge on a typical electron, $r$ is its distance from the point $(x, y, z), v$ is its velocity, $\left(v_{x}, v_{y}, v_{x}\right)$ the components of $v,(v, r)$ the angle between the direction of $v$ and $r$, and the bars over the letters mean that the position of the electron considered is that which it occupied at a time $t-\bar{r} / c$; and the summation is taken over all the electrons. We shall assume throughout the paper that the velocities of all the electrons are less than the velocity of radiation.

\section{Introduction and Evaluation of the two Scalar Potentials.}

Now let $x^{\prime}(t), y^{\prime}(t), z^{\prime}(t)$ denote the position of the electron $e$ at time $t$; and let $\bar{x}^{\prime}$ be used to denote $x^{\prime}(t-\bar{r} / r)$, so that $\bar{x}^{\prime}, \bar{y}^{\prime}, \bar{z}^{\prime}$ are known 
functions of $x, y, z, t$, when the motions of the electrons are known; we have $\vec{r}^{2}=\left(\bar{x}^{\prime}-x\right)^{2}+\left(\bar{y}^{\prime}-y\right)^{2}+\left(\bar{z}^{\prime}-z\right)^{2}$, and therefore

$$
\begin{gathered}
\bar{r} \frac{\partial \bar{r}}{\partial x}=-\left(\bar{x}^{\prime}-x\right)-\left\{\left(\bar{x}^{\prime}-x\right) v_{x}+\left(\bar{y}^{\prime}-y\right) v_{y}+\left(\bar{z}^{\prime}-z\right) v_{z}, \frac{1}{c} \frac{\partial \bar{r}}{\partial x}\right. \\
\frac{\partial \bar{r}}{\partial x}=-\frac{c\left(\bar{x}^{\prime}-x\right)}{c \bar{r}+\bar{r} \bar{v} \cos (\bar{v}, \bar{r})} .
\end{gathered}
$$

or

More generally, if $f$ be any function of the three quantities $\bar{x}^{\prime}-x, \bar{y}^{\prime}-y$, $\bar{z}^{\prime}-z$, and if $f_{1}, f_{2}, f_{3}$ denote its derivates with respect to these three arguments respectively, we easily find that

$$
\frac{\partial f}{\partial x}=-f_{1}+\frac{\left(x^{\prime}-x\right)\left(f_{1} \bar{v}_{x}+f_{2} \bar{v}_{y}+f_{\mathrm{s}} \bar{v}_{z}\right)}{c \bar{r}+\bar{r} \bar{v} \cos (\bar{v}, \bar{v})} .
$$

Similar equations hold for $\partial f / \partial y$ and $\partial f / \partial z$, while $\partial f / \partial t$ is given by the equation

$$
\frac{\partial f}{\partial t}=\frac{c\left(f_{1} \bar{x}_{x}+f_{2} \bar{v}_{y}+f_{3} \bar{v}_{z}\right)}{c+\bar{v} \cos (\bar{v}, \bar{v})} .
$$

Now define functions $F, G, \psi$ by the equations

$$
\begin{aligned}
& F(x, y, z, t)=\Sigma \frac{e}{4 \pi} \sinh ^{-1} \frac{\bar{z}^{\prime}-z}{\left\{\left(\bar{x}^{\prime}-x\right)^{2}+\left(\bar{y}^{\prime}-y\right)^{2}\right\}^{3}}, \\
& G(x, y, z, t)=\Sigma \frac{e}{4 \pi} \tan ^{-1} \frac{\bar{y}^{\prime}-y}{\bar{x}^{\prime}-x}, \\
& \psi(x, y, z, t)=\Sigma \frac{e}{4 \pi} \log \left\{\left(\bar{x}^{\prime}-x\right)^{2}+\left(\bar{y}^{\prime}-y\right)^{2}\right\}^{2},
\end{aligned}
$$

where the summation is taken over all the electrons. Using the formulæe just obtained for the derivates of a function of the kind $f$, we find that

$$
\begin{aligned}
\frac{\partial \psi}{\partial x}-\frac{\partial G}{\partial y} & =\Sigma \frac{e}{4 \pi} \frac{\bar{v}_{x}}{c \bar{r}+\bar{r} \bar{v} \cos (\bar{v}, \bar{r})}, \\
\frac{\partial \psi}{\partial y}+\frac{\partial G}{\partial x} & =\Sigma \frac{e}{4 \pi} \frac{\bar{v}_{y}}{c \bar{r}+\bar{r} \bar{v} \cos (\bar{v}, \bar{r})}, \\
\frac{\partial \psi}{\partial z}+\frac{1}{c} \frac{\partial F}{\partial t} & =\Sigma \frac{e}{4 \pi} \frac{\bar{v}_{z}}{c \bar{r}+\bar{r} \bar{v} \cos (\bar{v}, \bar{r})}, \\
\frac{1}{c} \frac{\partial \psi}{\partial t}+\frac{\partial F}{\partial z} & =-\Sigma \frac{e c}{4 \pi} \frac{1}{c \bar{r}+\bar{r} \bar{v} \cos (\bar{v}, \bar{r})}
\end{aligned}
$$

SEK. 2. VOL. 1. No. 843 . 
Combining these results with the expressions already found for $\phi, a_{x}, a_{y}$, $a_{\mathrm{z}}$, we have

$$
\begin{array}{cl}
\frac{\partial \psi}{\partial x}-\frac{\partial G}{\partial y}=a_{i x}, & \frac{\partial \psi}{\partial y}+\frac{\partial G}{\partial x}=a_{y}, \quad \frac{\partial \psi}{\partial z}+\frac{1}{c} \frac{\partial F}{\partial t}=a_{z}, \\
\frac{1}{c} \frac{\partial \psi}{\partial t}+\frac{\partial F}{\partial z}=-\phi .
\end{array}
$$

Substituting these results for $\phi, a_{x}, a_{y}, a_{z}$ in the equations of the type

$$
d_{x}=-\frac{1}{c} \frac{\partial a_{x}}{\partial t}-\frac{\partial \phi}{\partial x}, \quad h_{x}=\frac{\partial a_{z}}{\partial y}-\frac{\partial a_{y}}{\partial z},
$$

which give the components of the dielectric displacement and the magnetic force, we find that $\psi$ disappears automatically, and we obtain

$$
\begin{array}{ll}
d_{x}=\frac{\partial^{2} F}{\partial x \partial z}+\frac{1}{c} \frac{\partial^{2} G}{\partial y \partial t}, & d_{y}=\frac{\partial^{2} F}{\partial y \partial z}-\frac{1}{c} \frac{\partial^{2} G}{\partial x \partial t}, \quad d_{z}=\frac{\partial^{2} F}{\partial z^{2}}-\frac{1}{c^{2}} \frac{\partial^{2} F}{\partial t^{2}} ; \\
h_{x}=\frac{1}{c} \frac{\partial^{2} F}{\partial y \partial t}-\frac{\partial^{2} G}{\partial x \partial z}, & h_{y}=-\frac{1}{c} \frac{\partial^{2} F}{\partial x \partial t}-\frac{\partial^{2} G}{\partial y \partial z}, \quad h_{z}=\frac{\partial^{2} G}{\partial x^{2}}+\frac{\partial^{2} G}{\partial y^{2}} .
\end{array}
$$

These equations show that the six components of the dielectric displacement and the magnetic force can be expressed in terms of the derivates of two scalar potentials $F$ and $G$, defined by the equations

$$
\begin{aligned}
& F(x, y, z, t)=\Sigma \frac{e}{4 \pi} \sinh ^{-1} \frac{\bar{z}^{\prime}-z}{\left\{\left(\bar{x}^{\prime}-x\right)^{2}+\left(\bar{y}^{\prime}-y\right)^{2}\right\}^{2}}, \\
& G(x, y, z, t)=\Sigma \frac{e}{4 \pi} \tan ^{-1} \frac{\bar{y}^{\prime}-y}{\bar{x}^{\prime}-x},
\end{aligned}
$$

where the summation is taken over all the electrons in the field.

It can without difficulty be shown that, if any number of electrons whose total charge is zero are moving in any manner so as to remain always in the vicinity of a given point (i.e., to be in stationary motion), then the electromagnetic field thus generated is of the type given by

$$
F=\frac{1}{r} f\left(t-\frac{r}{c}\right), \quad G=0,
$$

where $r$ is the distance from the point and $f$ is an arbitrary function; or, more generally, of a field of this type superposed on fields of the same type, but related to the axes of $y$ and $x$ in the same way as this is related to the axis of $z$. This is perhaps of some interest in connection with the view advocated by some physicists that the atoms of the chemical elements consist of sets of electrons, whose total charge is zero, in stationary motion. 


\section{Discission of the Apparent Asymmetry of the preceding Result, and its Vector Expression.}

The formula thus obtained are not symmetrical with respect to $x, y$, and $z$. In order to discuss their relation to symmetrical formulæ, we observe that they can be written in the form of vector equations

$$
d=\operatorname{curl} \operatorname{curl} f+\operatorname{curl} \frac{1}{c} \dot{g}, \quad h=\operatorname{curl} \frac{1}{c} \dot{f}-\operatorname{curl} \text { curl } g,
$$

where $d$ and $h$ are the electric and magnetic vectors, and $f$ and $g$ are vectors directed parallel to the axis of $z$, whose magnitudes are $F$ and $G$ respectively. These vector equations are quite symmetrical, and our result is that, if, instead of regarding the electromagnetic field as defined by the vectors $d$ and $h$, we regard it as defined by vectors $f$ and $g$, connected with $d$ and $h$ by the above vector equations, then $f$ and $g$ are simple functions of the coordinates of the electrons, whereas $d$ and $h$ are complicated functions of their velocities and accelerations; and we have also obtained the result that without loss of generality we can take $f$ and $g$ to be everywhere, and at all times, parallel to some fixed direction in space (e.g., the axis of $z$ ), a fact which makes it possible to specify them by two scalar quantities only.

It might be asked whether vectors $f$ and $g$ exist which satisfy the above vector equations and which are perfectly symmetrical一the answer to this is in the negative; in fact, although the equations are themselves invariantive, and can therefore be expressed in the vector notation, yet they do not possess invariant solutions; just as the vector equation

$$
\operatorname{grad}\left(\frac{1}{r}\right)=\operatorname{curl} a
$$

(where $r$ is the scalar distance from the origin and $a$ is a rector to be (letermined) possesses an infinite number of solutions $a$, which can readily be found, but each of which is specially related to some line in space, so that no solution is symmetrical.

\section{Deduction of the General Functional Form of an Electrodynamic Disturbance in the स्Ether.}

Having now shown that an electrodynamic field due to electrons is completely characterized by two scalar potential functions $F^{\prime}$ and $G$, we can proceed to deduce its general functional form.

The functions $F$ and $G$ have singularities at those points which are actually occupied by electrons; at all other points we find by direct 
differentiation, or by substituting in the original electrodynamic equations the values of the components of dielectric displacement and magnetic force in terms of $F$ and $G$, that $F$ and $G$ satisfy the partial differential equations

$$
\begin{aligned}
& \frac{\partial^{2} F}{\partial x^{2}}+\frac{\partial^{2} F}{\partial y^{2}}+\frac{\partial^{2} F}{\partial z^{2}}-\frac{1}{c^{2}} \frac{\partial^{2} F}{\partial t^{2}}=0 \\
& \frac{\partial^{2} G}{\partial x^{2}}+\frac{\partial^{2} G}{\partial y^{2}}+\frac{\partial^{2} G}{\partial z^{2}}-\frac{1}{c^{2}} \frac{\partial^{2} G}{\partial t^{2}}=0 .
\end{aligned}
$$

Writing down the general solution of these latter equations," we obtain the result that the most general type of electrodynamic disturbance at a place in the ather not occupied by an electron is that for which the components of the dielectric displacement and magnetic force are represented by the equations

$$
\begin{array}{lll}
d_{x}=\frac{\partial^{2} F}{\partial x \partial z}+\frac{1}{c} \frac{\partial^{2} G}{\partial y \partial t}, & d_{y}=\frac{\partial^{2} F}{\partial y \partial z}-\frac{1}{c} \frac{\partial^{2} G}{\partial x \partial t}, & d_{z}=\frac{\partial^{2} F}{\partial z^{2}}-\frac{1}{c^{2}} \frac{\partial^{2} F}{\partial t^{2}} ; \\
h_{x}=\frac{1}{c} \frac{\partial^{2} F}{\partial y \partial t}-\frac{\partial^{2} G}{\partial x \partial z}, & h_{y}=-\frac{1}{c} \frac{\partial^{2} F}{\partial x \partial t}-\frac{\partial^{2} G}{\partial y \partial z}, & h_{z}=\frac{\partial^{2} G}{\partial x^{2}}+\frac{\partial^{2} G}{\partial y^{2}},
\end{array}
$$

where

$$
\begin{aligned}
& F=\int_{0}^{\pi} \int_{0}^{2 \pi} f(x \sin u \cos v+y \sin u \sin v+z \cos u+c t, u, v) d u d v, \\
& G=\int_{0}^{\pi} \int_{0}^{2 \pi} g(x \sin u \cos v+y \sin u \sin v+z \cos u+c t, u, v) d u d v,
\end{aligned}
$$

and $f$ and $g$ are arbitrary functions of their arguments.

* Cf. a paper by the author in Math. Ann., Vol. Lvrr., pp. 333-355, 1903. 